\title{
ABELIAN EXTENSIONS OF GLOBAL FIELDS WITH CONSTANT LOCAL DEGREE
}

\author{
HeRshy Kisilevsky And JaCK Sonn
}

\begin{abstract}
We prove that, given a global field $K$ and a positive integer $n$, there exists an abelian extension $L / K$ (of exponent $n$ ) such that the local degree of $L / K$ is equal to $n$ at every finite prime of $K$, and is equal to two at the real primes if $n=2$. As a consequence, we prove that the $n$-torsion subgroup of the Brauer group of $K$ is equal to the relative Brauer group of $L / K$.
\end{abstract}

\section{Introduction}

Let $K$ be a field, $\operatorname{Br}(K)$ its Brauer group. If $L / K$ is a field extension, then the relative Brauer group $\operatorname{Br}(L / K)$ is the kernel of the restriction map $\operatorname{res}_{L / K}$ : $B r(K) \rightarrow B r(L)$. Relative Brauer groups have been studied by Fein and Schacher (see e.g. [4,5,6].) Every subgroup of $\operatorname{Br}(K)$ is a relative Brauer group $\operatorname{Br}(L / K)$ for some extension $L / K[4]$, and the question arises as to which subgroups of $B r(K)$ are algebraic relative Brauer groups, i.e. of the form $\operatorname{Br}(L / K)$ with $L / K$ an algebraic extension. For example if $L / K$ is a finite extension of number fields, then $\operatorname{Br}(L / K)$ is infinite [5], so no finite subgroup of $\operatorname{Br}(K)$ is an algebraic relative Brauer group. In [2] the question was raised as to whether or not the $n$-torsion subgroup $B r_{n}(K)$ of the Brauer group $\operatorname{Br}(K)$ of a field $K$ is an algebraic relative Brauer group. For example, if $K$ is a (p-adic) local field, then $B r(K) \cong \mathbb{Q} / \mathbb{Z}$, so $B r_{n}(K)$ is an algebraic relative Brauer group for all $n$. A counterexample was given in [2] for $n=2$ and $K$ a formal power series field over a local field. For global fields $K$, the problem is a purely arithmetic one, because of the fundamental local-global description of the Brauer group of a global field. In particular, for a Galois extension $L / K$ of global fields, if the local degree of $L / K$ at every finite prime is equal to $n$, and is equal to 2 at the real primes for $n$ even, then $B r(L / K)=B r_{n}(K)$. In [2], it was proved that $B r_{n}(\mathbb{Q})$ is an algebraic relative Brauer group for all squarefree $n$. In [8], the arithmetic criterion above was verified for any number field $K$ Galois over $\mathbb{Q}$ and any $n$ prime to the class number of $K$, so in particular, $B r_{n}(\mathbb{Q})$ is an algebraic relative Brauer group for all $n$. In [9], Popescu proved that for a global function field $K$ of characteristic $p$, the arithmetic criterion holds for $n$ prime to the order of the non- $p$ part of the Picard group of $K$.

In this paper we settle the question completely, by verifying the arithmetic criterion for all $n$ and all global fields $K$. In particular, the $n$-torsion subgroup of the Brauer

Received by the editors January 31, 2005.

The research of the first author was supported by NSERC and FQRNT. The research of the second author was supported by the VPR Fund at the Technion. 
group of $K$ is an algebraic relative Brauer group for all $n$ and all global fields $K$. The proof, an extension of the ideas in [8], reduces to the case $n$ a prime power $\ell^{r}$. We first carry out the proof for number fields $K$. The proof for the function field case when $\ell \neq \operatorname{char}(K)$ is essentially the same as the proof in the number field case. The proof for $\ell=\operatorname{char}(K)$ appears in [9].

\section{A splitting lemma}

Let $K$ be a number field, $\mathfrak{p}$ a finite prime of $K, I_{\mathfrak{p}}$ the group of fractional ideals prime to $\mathfrak{p}, P_{\mathfrak{p}}$ the group of principal fractional ideals in $I_{\mathfrak{p}}, P_{\mathfrak{p}, 1}$ the group of principal fractional ideals in $P_{\mathfrak{p}}$ generated by elements congruent to $1 \bmod \mathfrak{p}$. Then $C l_{K} \cong$ $I_{\mathfrak{p}} / P_{\mathfrak{p}}$ is the class group of $K, C l_{K, \mathfrak{p}} \cong I_{\mathfrak{p}} / P_{\mathfrak{p}, 1}$ is the ray class group with conductor $\mathfrak{p}$, and $\bar{P}_{\mathfrak{p}}=P_{\mathfrak{p}} / P_{\mathfrak{p}, 1}$ is the principal ray with conductor $\mathfrak{p}$. We have a short exact sequence

$$
1 \longrightarrow \bar{P}_{\mathfrak{p}} \longrightarrow C l_{K, \mathfrak{p}} \longrightarrow C l_{K} \longrightarrow 1
$$

Let $\ell$ be a prime dividing the orders of all three terms of $(*)$, and consider the exact sequence of $\ell$-primary components

$$
1 \longrightarrow \bar{P}_{\mathfrak{p}}^{(\ell)} \longrightarrow C l_{K, \mathfrak{p}}^{(\ell)} \longrightarrow C l_{K}^{(\ell)} \longrightarrow 1
$$

We are interested in primes $\mathfrak{p}$ for which the sequence $\left({ }^{*} \ell\right)$ splits. Let $\mathfrak{a}_{1}, \ldots, \mathfrak{a}_{s} \in I_{\mathfrak{p}}$ such that their images $\overline{\mathfrak{a}}_{i}$ in $C l_{K}^{(\ell)}$ form a minimal set of generators of the finite abelian $\ell$-group $C l_{K}^{(\ell)}$. Let $\ell^{m_{i}}$ be the order of $\overline{\mathfrak{a}}_{i}, i=1, \ldots, s$. Then $\mathfrak{a}_{i}^{\ell^{m_{i}}}=\left(a_{i}\right) \in P_{\mathfrak{p}}$, $i=1, \ldots, s$. Let $K_{1}=K\left(\sqrt[\ell^{m}]{a_{i}} \mid 1 \leq i \leq s\right) K\left(\mu_{\ell^{m}}\right)$ with $m=\max \left\{m_{1}, \ldots, m_{s}\right\}$.

Lemma 2.1. In order that the sequence $\left({ }_{\ell}^{*}\right)$ split, it is sufficient that $\mathfrak{p}$ split completely in $K_{1}$.

Proof. Suppose $\mathfrak{p}$ splits in $K_{1}$. Then $a_{i}$ is locally an $\ell^{m_{i}}$-th power at $\mathfrak{p}$, so there exists $\alpha_{i} \in K^{*}$ such that $\alpha_{i}^{\ell^{m_{i}}} \equiv a_{i}(\bmod \mathfrak{p}), i=1, \ldots, s$. Set $\mathfrak{b}_{i}:=\mathfrak{a}_{i} \alpha_{i}^{-1}, i=1, \ldots, s$. Then $\overline{\mathfrak{b}}_{i}=\overline{\mathfrak{a}}_{i}, i=1, \ldots, s$, and $\mathfrak{b}_{i}^{\ell^{m_{i}}}=\mathfrak{a}_{i}^{\ell^{m_{i}}} \alpha_{i}^{-\ell^{m_{i}}}=\left(a_{i}\right) \alpha_{i}^{-\ell^{m_{i}}}$ with $a_{i} \alpha_{i}^{-\ell^{m_{i}}} \equiv 1(\bmod$ $\mathfrak{p})$, so $\mathfrak{b}_{i}^{\ell^{m_{i}}} \in P_{\mathfrak{p}, 1}$. Let $\widetilde{\mathfrak{b}}_{i}$ be the image of $\mathfrak{b}_{i}$ in $C l_{K, \mathfrak{p}}^{(\ell)}$. We have just seen that its order divides (in fact equals) $\ell^{m_{i}}$. Hence the $\widetilde{\mathfrak{b}}_{i}$ together generate a subgroup of order at most $\ell^{\sum m_{i}}$. Since the $\overline{\mathfrak{b}}_{i}=\overline{\mathfrak{a}}_{i}$ generated the $\ell$-class group $C l_{K}^{(\ell)}$, of order $\ell^{\sum m_{i}}$, it follows that the $\widetilde{\mathfrak{b}}_{i}$ together generate a complement to $\bar{P}_{\mathfrak{p}}^{(\ell)}$ in $C l_{K, \mathfrak{p}}^{(\ell)}$.

Note. In the proof of Lemma 2.1, we could have taken $\mathfrak{a}_{1}, \ldots, \mathfrak{a}_{s} \in I_{\mathfrak{p}}$ to be prime ideals outside of any given finite set of primes of $K$, by virtue of Chebotarev's density theorem. 


\section{3. $\ell^{r}$-torsion in the Brauer group}

Theorem 3.1. Let $K$ be a number field, $\ell$ a prime number, $r$ a positive integer. Then there exists an abelian extension $L / K$ of exponent $\ell^{r}$ such that the local degree $\left[L K_{\mathfrak{p}}: K_{\mathfrak{p}}\right]$ is equal to $\ell^{r}$ for every finite prime $\mathfrak{p}$ of $K$, and is equal to 2 at every real prime if $\ell=2$.

Proof. Let $H_{K}$ be the Hilbert class field of $K$, and let $H_{K}^{(\ell)}$ be the $\ell$-primary part of $H_{K}$. Let $\ell^{t}$ be the exponent of $C l_{K}^{(\ell)}$.

Let $S$ be the set of primes of $K$ that split completely in the extension $F$ of $K$ generated by $H_{K}^{(\ell)}$, all $\ell^{r+t}$-th roots of all units (including roots of unity) of $K$, and the field $K_{1}$ of Lemma 2.1. For $\mathfrak{p} \in S, \bar{P}_{\mathfrak{p}} \cong\left(\mathcal{O}_{K} / \mathfrak{p}\right)^{*}$ modulo the image of the unit group $E_{K}$ of $K$. By definition of $S$, every unit of $K$ is an $\ell^{r+t}$-th power in $K_{\mathfrak{p}}^{*}$, so the cyclic group $\bar{P}_{\mathfrak{p}}^{(\ell)}$ has order divisible by $\ell^{r+t}$. Let $R^{(\mathfrak{p}, \ell)}$ denote the $\ell$-ray class field with conductor $\mathfrak{p}$. It follows that the subfield of $R^{(\mathfrak{p}, \ell)}$ fixed by the $\ell^{t}$-torsion subgroup of $\operatorname{Gal}\left(R^{(\mathfrak{p}, \ell)} / K\right)$ is a cyclic extension of $K$ of degree divisible by $\ell^{r}$. Let $L^{\mathfrak{p}}$ be the subfield of this field of degree exactly $\ell^{r}$ over $K . L^{\mathfrak{p}} / K$ is totally ramified at $\mathfrak{p}$ and $\mathfrak{p}$ is the only prime of $K$ ramifying in $L^{\mathfrak{p}}$.

For each $\mathfrak{p} \in S$ the splitting of the short exact sequence $\left(*_{\ell}\right)$ allows us to fix a splitting map $f_{\mathfrak{p}}: C l_{K, \mathfrak{p}}^{(\ell)} \longrightarrow \bar{P}_{\mathfrak{p}}^{(\ell)}$.

By [3, Theorem 5, p. 105], there exists a cyclic extension $L_{0} / K$ of degree $\ell^{r}$ having local degree $\ell^{r}$ at the primes $\mathfrak{l}_{1}, \ldots, \mathfrak{l}_{g}$ dividing $\ell$, and local degree 2 at the real primes if $\ell=2$.

Let $\mathfrak{q}_{1}, \ldots, \mathfrak{q}_{e}$ be the primes of $K$ not dividing $\ell$, which ramify in $L_{0}$, ordered in such a way that $L_{0} / K$ has local degree $\ell^{r}$ at $\mathfrak{q}_{1}, \ldots, \mathfrak{q}_{d}, 0 \leq d \leq e$, and $L_{0} / K$ has local degree less than $\ell^{r}$ at $\mathfrak{q}_{d+1}, \ldots, \mathfrak{q}_{e}$.

Using the Note following the proof of Lemma 2.1, we may assume without loss of generality that the ideals $\mathfrak{a}_{1}, \ldots, \mathfrak{a}_{s}$ in Lemma 2.1, representing the elements of the class group, are prime ideals, distinct from $\mathfrak{l}_{1}, \ldots, \mathfrak{l}_{g}$ and from $\mathfrak{q}_{1}, \ldots, \mathfrak{q}_{e}$.

Suppose $d<e$. Let $\mathfrak{q}=\mathfrak{q}_{d+1}$, and let $\ell^{a}, \ell^{b}$ be the ramification index and inertia degree respectively of $\mathfrak{q}$ in $L_{0} / K$.

We seek a prime $\mathfrak{p} \in S$ such that

(1) $\mathfrak{p}$ splits completely in $L_{0}$,

(2) $\mathfrak{l}_{1}, \ldots, \mathfrak{l}_{g}$ and $\mathfrak{q}_{1}, \ldots, \mathfrak{q}_{d}$ split completely in $L^{\mathfrak{p}}$, and

(3) the local degree of $L_{0} L^{\mathfrak{p}} / K$ at $\mathfrak{q}$ is $\ell^{r}$.

(1) is a Chebotarev splitting condition compatible with the Chebotarev condition $\mathfrak{p} \in S$. (2) is equivalent to a Chebotarev splitting condition on $\mathfrak{p}$, by a "reciprocity argument" (cf. [8]), formulated in the following lemma.

Lemma 2.2. Let $\mathfrak{a}$ be any prime of $K$, and let $\mathfrak{p}$ be any prime in $S$ different from $\mathfrak{a}$. Let $L^{\prime}$ be a subfield of $L^{\mathfrak{p}}$ containing $K$, of degree $\ell^{s}$ over $K$. Let $\ell^{m}$ be the order of the image of $\mathfrak{a}$ in $C l_{K}^{(\ell)}$, and let $\mathfrak{a}^{\ell^{m}}=(\alpha) \in P_{\mathfrak{p}}$. Then $\mathfrak{a}$ splits completely in $L^{\prime}$ if and only if $\mathfrak{p}$ splits completely in $K\left(\mu_{\ell^{m+s}}, \sqrt[\ell^{m+s}]{\alpha}\right)$. 
Proof. Let $\widetilde{\beta})=f_{\mathfrak{p}}(\tilde{\mathfrak{a}}) \in \bar{P}_{\mathfrak{p}}^{(\ell)}$, where $f_{\mathfrak{p}}: C l_{K, \mathfrak{p}}^{(\ell)} \longrightarrow \bar{P}_{\mathfrak{p}}^{(\ell)}$ is the splitting map above, and $\tilde{\mathfrak{a}}$ denotes the image of $\mathfrak{a}$ in $C l_{K, \mathfrak{p}}^{(\ell)}$. Then

$$
\widetilde{(\beta)} \ell^{\ell^{m}}=f_{\mathfrak{p}}(\tilde{\mathfrak{a}})^{\ell^{m}}=f_{\mathfrak{p}}\left(\tilde{\mathfrak{a}}^{\ell^{m}}\right)=f_{\mathfrak{p}}(\widetilde{(\alpha)})=\widetilde{(\alpha)}
$$

If $\mathfrak{p} \in S$, the cyclic group $\bar{P}_{\mathfrak{p}}^{(\ell)}$ has order divisible by $\ell^{r+t}$ hence by $\ell^{m+s}$ (because $m \leq t$ and $s \leq r)$. Now $\mathfrak{a}$ splits completely in $L^{\prime}$ if and only if the Frobenius of $\mathfrak{a}$ in $\operatorname{Gal}\left(L^{\prime} / K\right)$ is trivial, which holds if and only if $\left.\widetilde{\beta}\right)=f_{\mathfrak{p}}(\tilde{\mathfrak{a}})$ is an $\ell^{s}$ th power in $\bar{P}_{\mathfrak{p}}^{(\ell)}$, which holds if and only if $\widetilde{(\alpha)}=\widetilde{(\beta)}{ }^{\ell^{m}}$ is an $\ell^{m+s}$ th power in $\bar{P}_{\mathfrak{p}}^{(\ell)}$ (since $\bar{P}_{\mathfrak{p}}^{(\ell)}$ has order divisible by $\ell^{m+s}$, which holds if and only if $\alpha$ is an $\ell^{m+s}$ th power $\bmod \mathfrak{p}$, which holds if and only if the polynomial $x^{\ell^{m+s}}-\alpha$ has a root mod $\mathfrak{p}$, which holds if and only if $x^{\ell^{m+s}}-\alpha$ factors into linear factors $\bmod \mathfrak{p}\left(\mathfrak{p}\right.$ splits completely in $K\left(\mu_{\ell^{r+t}}\right)$ ), which holds if and only if $\mathfrak{p}$ splits completely in $K\left(\mu_{\ell^{m+s}}, \sqrt[\ell^{m+s}]{\alpha}\right)$.

It follows from the Lemma that the second condition is a Chebotarev splitting condition on $\mathfrak{p}$, hence compatible with the previous conditions. The third condition, that the local degree of $L_{0} L^{\mathfrak{p}} / K$ at $\mathfrak{q}$ is $\ell^{r}$, will hold if $\mathfrak{q}$ is unramified in $L^{\mathfrak{p}} / K$ with degree of inertia exactly $\ell^{r-a}$. This condition is equivalent, by Lemma 2.2 , to the condition that $\mathfrak{p}$ splits completely in $K\left(\mu_{\ell^{m+a}}, \sqrt[\ell^{m+a}]{\alpha}\right)$ but not in $K\left(\mu_{\ell^{m+a+1}}, \sqrt[\ell^{m+a+1}]{\alpha}\right)$, where $\mathfrak{q}$ plays the role of $\mathfrak{a}$ in Lemma 2.2. $\ell^{m}$ is the order of $\mathfrak{q}$ in the class group. Note that $a<r$ here, so $m+a+1 \leq r+t$. In order that this last Chebotarev condition be compatible with the preceding ones, it is necessary and sufficient that $\sqrt[e^{m+a+1}]{\alpha}$ not lie in the composite of all the fields in which $\mathfrak{p}$ has been required to split. This follows from ramification considerations, since the ramification index of $\mathfrak{q}$ in the composite of all the fields in which $\mathfrak{p}$ has been required to split, is exactly $\ell^{a}$, whereas the ramification index of $\mathfrak{q}$ in $K\left(\mu_{\ell^{m+a+1}}, \sqrt[\ell^{m+a+1}]{\alpha}\right)$ is $\ell^{a+1}$. We have therefore proved the existence of a prime (in fact infinitely many) $\mathfrak{p}=\mathfrak{p}_{1}$ satisfying the three conditions.

Similarly, there exists a prime $\mathfrak{p}_{2} \in S$, such that

(4) $\mathfrak{p}_{2}$ splits completely in $L_{0}$ and $L_{1}:=L^{\mathfrak{p}_{1}}$,

(5) $\mathfrak{l}_{1}, \ldots, \mathfrak{l}_{g}, \mathfrak{q}_{1}, \ldots, \mathfrak{q}_{d}, \mathfrak{q}_{d+1}, \mathfrak{p}_{1}$ split completely in $L_{2}:=L^{\mathfrak{p}_{2}}$, and

(6) the local degree of $L_{0} L^{\mathfrak{p}_{2}} / K$ at $\mathfrak{q}_{d+2}$ is $\ell^{r}$.

Proceeding in this manner, we get $\mathfrak{p}_{1}, \ldots, \mathfrak{p}_{e-d}$, such that

(7) $\mathfrak{p}_{i}$ splits completely in $L_{j}$ for $i \neq j$,

(8) $\mathfrak{l}_{1}, \ldots, \mathfrak{l}_{g}, \mathfrak{q}_{1}, \ldots, \mathfrak{q}_{e}$ split completely in $L_{1}, \ldots, L_{e-d}$, and

(9) the local degree of the composite $L_{0} L_{1} \cdots L_{e-d}$ is equal to $\ell^{r}$ at each of the primes $\mathfrak{l}_{1}, \ldots, \mathfrak{l}_{g}, \mathfrak{q}_{1}, \ldots, \mathfrak{q}_{e}, \mathfrak{p}_{1}, \ldots, \mathfrak{p}_{e-d}$.

Let us now extend $\mathfrak{l}_{1}, \ldots, \mathfrak{l}_{g}, \mathfrak{q}_{1}, \ldots, \mathfrak{q}_{e}, \mathfrak{p}_{1}, \ldots, \mathfrak{p}_{e-d}$ to an enumeration

$$
\mathfrak{l}_{1}, \ldots, \mathfrak{l}_{g}, \mathfrak{q}_{1}, \ldots, \mathfrak{q}_{e}, \mathfrak{p}_{1}, \ldots, \mathfrak{p}_{e-d}, \mathfrak{a}_{1}, \mathfrak{a}_{2}, \ldots, \mathfrak{a}_{s}, \mathfrak{a}_{s+1}, \ldots
$$

of all the finite primes of $K$, where $\mathfrak{a}_{1}, \mathfrak{a}_{2}, \ldots, \mathfrak{a}_{s}$ are the primes of Lemma 2.1.

We now seek a prime $\mathfrak{p}=\mathfrak{p}_{e-d+1} \in S$ such that 
(10) $\mathfrak{p}$ splits completely in $L_{0}, L_{1}, \ldots, L_{e-d}$,

(11) $\mathfrak{l}_{1}, \ldots, \mathfrak{l}_{g}, \mathfrak{q}_{1}, \ldots, \mathfrak{q}_{e}, \mathfrak{p}_{1}, \ldots, \mathfrak{p}_{e-d}$ split completely in $L^{\mathfrak{p}}$, and

(12) $\mathfrak{a}=\mathfrak{a}_{1}$ is inert in $L^{\mathfrak{p}}$.

(10) and (11) are equivalent to saying that $\mathfrak{p}$ splits completely in a Galois extension (a composite of $L_{i}$ 's and fields used in proving compatibility of previous Chebotarev conditions) in which $\mathfrak{a}$ is unramified. (12) is equivalent to the condition that $\mathfrak{a}$ does not split completely in the subfield of $L^{\mathfrak{p}}$ of degree $\ell$ over $K$. By Lemma 2.2 applied to this $\mathfrak{a}$, this condition is equivalent to the (Chebotarev) condition that $\mathfrak{p}$ does not split completely in $K\left(\mu_{\ell^{m+1}}, \sqrt[\ell^{m+1}]{\alpha}\right)$, where $m$ corresponds to this $\mathfrak{a}$. Since $\mathfrak{a}$ ramifies in $K\left(\mu_{\ell^{m+1}}, \sqrt[\ell^{m+1}]{\alpha}\right)$, this last Chebotarev condition is compatible with the others.

We remove $\mathfrak{p}=\mathfrak{p}_{e-d+1}$ from the enumeration, denote $L^{\mathfrak{p}}$ by $L_{e-d+1}$, and move to the next ideal $\mathfrak{a}_{2}$. A similar argument yields a prime $\mathfrak{p}=\mathfrak{p}_{e-d+2}$ and a corresponding $L_{e-d+2}$, such that

(13) $\mathfrak{p}$ splits completely in $L_{0}, L_{1}, \ldots, L_{e-d+1}$,

(14) $\mathfrak{l}_{1}, \ldots, \mathfrak{l}_{g}, \mathfrak{q}_{1}, \ldots, \mathfrak{q}_{e}, \mathfrak{p}_{1}, \ldots, \mathfrak{p}_{e-d+1}$ split completely in $L^{\mathfrak{p}}$, and

(15) $\mathfrak{a}=\mathfrak{a}_{2}$ is inert in $L^{\mathfrak{p}}=L_{e-d+2}$.

Continuing in this way, a sequence $\left\{\mathfrak{p}_{i}\right\}$ of primes of $K$ is generated, with a corresponding sequence of fields $\left\{L_{i}\right\}$.

Form the composite $L:=L_{0} L_{1} L_{2} \cdots$. The local degree of $L / K$ at every finite prime is $\ell^{r}$. In fact, the local degree of $L_{0} L_{1} \cdots L_{e-d}$ is equal to $\ell^{r}$ at each of the primes $\mathfrak{l}_{1}, \ldots, \mathfrak{l}_{g}, \mathfrak{q}_{1}, \ldots, \mathfrak{q}_{e}, \mathfrak{p}_{1}, \ldots, \mathfrak{p}_{e-d}$, as we showed earlier; these primes split completely in $L_{j}$ for $j>e-d$; at every $\mathfrak{p}_{i}$, the local extension is cyclic and totally ramified of degree $\ell^{r}$; and at every $\mathfrak{a} \notin\left\{\mathfrak{l}_{1}, \ldots, \mathfrak{l}_{g}, \mathfrak{q}_{1}, \ldots, \mathfrak{q}_{e}, \mathfrak{p}_{1}, \mathfrak{p}_{2}, \ldots\right\}$, the local extension is cyclic and unramified of degree $\ell^{r}$. The local degree at the real primes is 2 since this is true already for $L_{0}$.

\section{The function field case.}

Let $K$ be a global function field of characteristic $p$. Let $\ell$ be a prime different from $p$. Theorem 3.1 holds also for $K$, with essentially the same proof, except simpler, since there are no primes dividing $\ell$. We indicate how this works.

By Chebotarev's density theorem for function fields (see e.g. [10]), there exists a prime $\mathfrak{p}$ which is inert in the constant degree $\ell$ extension of $K$. This property is equivalent to $\mathfrak{p}$ having degree prime to $\ell$ (see e.g. [9]). Fix one such prime $\mathfrak{p}_{\infty}$. Let $\mathcal{O}$ be the ring of all elements of $K$ which are integral at all primes $\mathfrak{p} \neq \mathfrak{p}_{\infty}$ of $K$. $\mathcal{O}$ is a Dedekind domain. Call $\mathfrak{p}_{\infty}$ the infinite prime of $K$ and all the others finite primes of $K$. There is an exact sequence

$$
1 \longrightarrow \operatorname{Pic}^{0}(K) \longrightarrow \operatorname{Pic}(\mathcal{O}) \longrightarrow \mathbb{Z} / d_{\infty} \mathbb{Z} \longrightarrow 0
$$

with $\operatorname{Pic}^{0}(K)$ the group of divisor classes of degree zero of $K$, $\operatorname{Pic}(\mathcal{O})$ the ideal class group of $\mathcal{O}$, and the third arrow is the degree $\bmod d_{\infty}$ map, where $d_{\infty}$ denotes the degree of $\mathfrak{p}_{\infty}$. As $d_{\infty}$ and $\ell$ are relatively prime, the $\ell$-primary components of 
$\operatorname{Pic}^{0}(K)$ and $\operatorname{Pic}(\mathcal{O})$ are isomorphic; we denote them by $C l_{K}^{(\ell)}$. If $\mathfrak{p}$ is a finite prime of $K$, let $R^{(\mathfrak{p})}$ be the maximal abelian extension of $K$ with conductor dividing $\mathfrak{p}$, in which $\mathfrak{p}_{\infty}$ splits completely, i.e. the ray class field $\bmod \mathfrak{p} . \operatorname{Gal}\left(R^{(\mathfrak{p})} / K\right)$ is canonically isomorphic, via the reciprocity map, to $C l_{K, \mathfrak{p}}$, the ray class group mod $\mathfrak{p}$ (See $[7, \mathrm{p}$. 204].

The proofs of Lemma 2.1, Lemma 2.2 and Theorem 3.1 proceed as in the number field case, except that there are no primes $\mathfrak{l}_{1}, \ldots, \mathfrak{l}_{g}, \mathfrak{q}_{1}, \ldots, \mathfrak{q}_{e}$ to deal with; the field $L_{0}$ is replaced with the constant degree $\ell^{r}$ extension of $K$, and $e=0 . L$ is the composite of the fields $L_{0}, L_{1}, L_{2}, \ldots$. We therefore have

Theorem 4.1. Let $K$ be a global function field of characteristic $p, \ell$ a prime different from $p, r$ a positive integer. Then there exists an abelian extension $L / K$ of exponent $\ell^{r}$ such that the local degree $\left[L K_{\mathfrak{p}}: K_{\mathfrak{p}}\right]$ is equal to $\ell^{r}$ for every prime $\mathfrak{p}$ of $K$.

\section{The $n$-torsion subgroup of the Brauer group of $K$}

Theorem 5.1. Given a global field $K$ and a positive integer $n$, there exists an abelian extension $L / K$ (of exponent $n$ ) such that the $n$-torsion subgroup of the Brauer group of $K$ is equal to the relative Brauer group of $L / K$.

Proof. Consider the case $n=\ell^{r}, \ell$ prime. By Theorems 3.1 and 4.1, if $\operatorname{char}(K) \neq \ell$, there exists an abelian $\ell$-extension $L / K$ whose local degree at every nonarchimedian prime is equal to $\ell^{r}$, and is equal to 2 at the real primes if $K$ is a number field and $\ell=2$. If $\operatorname{char}(K)=\ell$, the same result holds by [9]. It follows from the fundamental theorem of class field theory on the Brauer group of a number field that $L$ splits every algebra class of order dividing $\ell^{r}$, and conversely, any algebra class split by $L$ has order dividing $\ell^{r}$. For general $n$, the theorem follows from a straightforward reduction to the prime power case (see [2]).

Remark. Adrian Wadsworth has pointed out to us that in the case char $(K)=\ell, B r_{\ell^{r}}(K)$ is the relative Brauer group of a purely inseparable extension, namely $L=\sqrt[\ell^{r}]{K}$, by results of Albert [1, p. 109].

\section{Acknowledgments}

We are grateful to Harold Stark for valuable discussions.

\section{References}

[1] A. A. Albert, Structure of Algebras, AMS, Providence, R.I., 1961.

[2] E. Aljadeff and J. Sonn, Relative Brauer groups and m-torsion, Proc. AMS 130 (2002), 13331337.

[3] E. Artin and J. Tate, Class Field Theory, W. A. Benjamin, New York, 1968.

[5] B. Fein, W. Kantor, and M. Schacher, Relative Brauer groups II, J. R. Ang. Math. 328 (1981), 39-57.

[4] B. Fein and M. Schacher, Relative Brauer groups I, J. R. Ang. Math. 321 (1981), 179-194.

[6] _ Relative Brauer groups III, J. R. Ang. Math. 335 (1982), 37-39.

[7] D. R. Hayes, Explicit class field theory in global function fields, in Studies in Algebra and Number Theory (G. C. Rota, Ed.) (1979), Academic Press, New York. 
[8] H. Kisilevsky and J. Sonn, On the $n$-torsion subgroup of the Brauer group of a number field, J. Theorie des Nombres de Bordeaux 15 (2003), 199-204.

[9] C. Popescu, Torsion subgroups of Brauer groups and extensions of constant local degree for global function fields, to appear in J. Number Theory.

[10] A. Weil, Basic Number Theory, Springer-Verlag, Berlin-New York, 1967.

Concordia University, Department of Mathematics and Statistics, 1455 de Maisonneuve Blvd. West, Montreal, Quebec H3G 1M8, Canada

E-mail address: kisilev@mathstat.concordia.ca

Technion-IsRael Institute of Technology, Faculty of Mathematics, 32000 Haifa, IsRael

E-mail address: sonn@math.technion.ac.il 